\title{
Worsening Autoimmune Neutropenia After Stopping Ibrutinib in a Patient With Chronic Lymphocytic Leukemia: Case Report and Review of Literature
}

\author{
Natalie Rosen, BA, Thomas Klumpp, MD, and Sameh Gaballa, MD, MSc
}

\section{INTRODUCTION}

Autoimmune cytopenia (AIC) is relatively common in patients with chronic lymphocytic leukemia occurring in $5-10 \%$ of patients during the course of their disease. ${ }^{1}$ Autoimmune hemolytic anemia (AlHA) constitutes the highest prevalence (5-10\%) of CLL-associated AIC followed by idiopathic thrombocytopenic purpura (ITP) $(2-5 \%)$, pure red cell aplasia (PRCA) $(<1 \%)$, and autoimmune neutropenia (AIN) $(<1 \%)^{2}{ }^{2.3}$ The prevalence of AIN, however, may in fact be higher than reported due to a lack of awareness of the condition and difficulty in its diagnosis. ${ }^{4}$ Despite its rarity, autoimmune neutropenia can be a significant clinical challenge in patients with CLL and can increase the risk of infectious complications. Thus, the prompt diagnosis and resolution of CLL-associated AIN is essential to the management of these patients.

Ibrutinib is a selective inhibitor of Bruton tyrosine kinase and induces a durable response in patients with CLL. ${ }^{5}$ The activity of ibrutinib in CLL-associated AIC is largely unknown as pivotal clinical trials excluded patients with AIC. We report a case of a patient with CLL who experienced worsening of AIN after discontinuing ibrutinib therapy. Given its immune modulatory effects, these findings suggest ibrutinib may have a role in controlling AIN in patients with CLL.

\section{CASE PRESENTATION}

This is a 72 year-old female who was diagnosed with CLL in early 2000 after presenting with progressive left cervical lymphadenopathy. Prognostic markers of CLL were not evaluated at the time of diagnosis. She was treated with fludarabine and rituximab and achieved a complete response. She continued on active surveillance for five years until she had evidence of disease recurrence.

She was treated with single agent rituximab in 2005 and then rituximab-bendamustine in 2009 and achieved complete remission. In 2012, she had evidence of disease progression with diffuse adenopathy and a biopsy was performed to rule out disease transformation. FISH studies at that time revealed trisomy 12 and $17 p$ deletion. The patient did not wish to undergo further chemotherapy and instead was treated with high dose methylprednisolone with rituximab followed by lenalidomide. Her CLL was controlled on this regimen, however, she developed thrombocytopenia (platelet count of 46,000 per cubic millimeter), leg heaviness, and fatigue from the lenalidomide and it was stopped in June 2014. Three weeks after stopping lenalidomide, she developed incidental neutropenia with an absolute neutrophil count (ANC) of 900 cells/ $/ \mathrm{L}$. Due to a lack of constitutional symptoms, she was not treated for her neutropenia and her counts improved spontaneously.

In September 2014, she was started on ibrutinib for disease progression and achieved a partial response. However, by August 2017 she noted increased pelvic lymphadenopathy and leukocytosis consistent with progressive disease. Genome sequencing studies revealed the presence of a BTKI mutation (C481S) conferring resistance to ibrutinib as well as an IGH-BCL2 rearrangement, ATM R248, and NOTCH1. Ibrutinib was discontinued in November 2017 and venetoclax was initiated. Subsequently, her ANC began declining which was initially thought to be a related side effect of venetoclax (Figure 1). However, despite multiple dose reductions and interrupting venetoclax for 7 weeks, her ANC remained below 500 cells/uL. The differential diagnosis at this time included myelodysplastic syndrome and autoimmune neutropenia. Therefore, a bone marrow biopsy was performed which revealed only $10 \%$ involvement with CLL and no evidence of myelodysplasia. Moreover, there was evidence of myeloid maturation arrest further suggesting autoimmunity as her etiology of neutropenia. She received oral steroids and rituximab which resulted in a rapid resolution of her neutropenia. However, due to a decreasing ANC (Figure 1), tbo-filgastrim was added to her regimen every 10 days. Venetoclax was restarted, in conjunction with tbo-filgastrim, and her ANC continued to be maintained above 1000 cells/uL. She has remained stable on this therapeutic regimen for almost one year. 


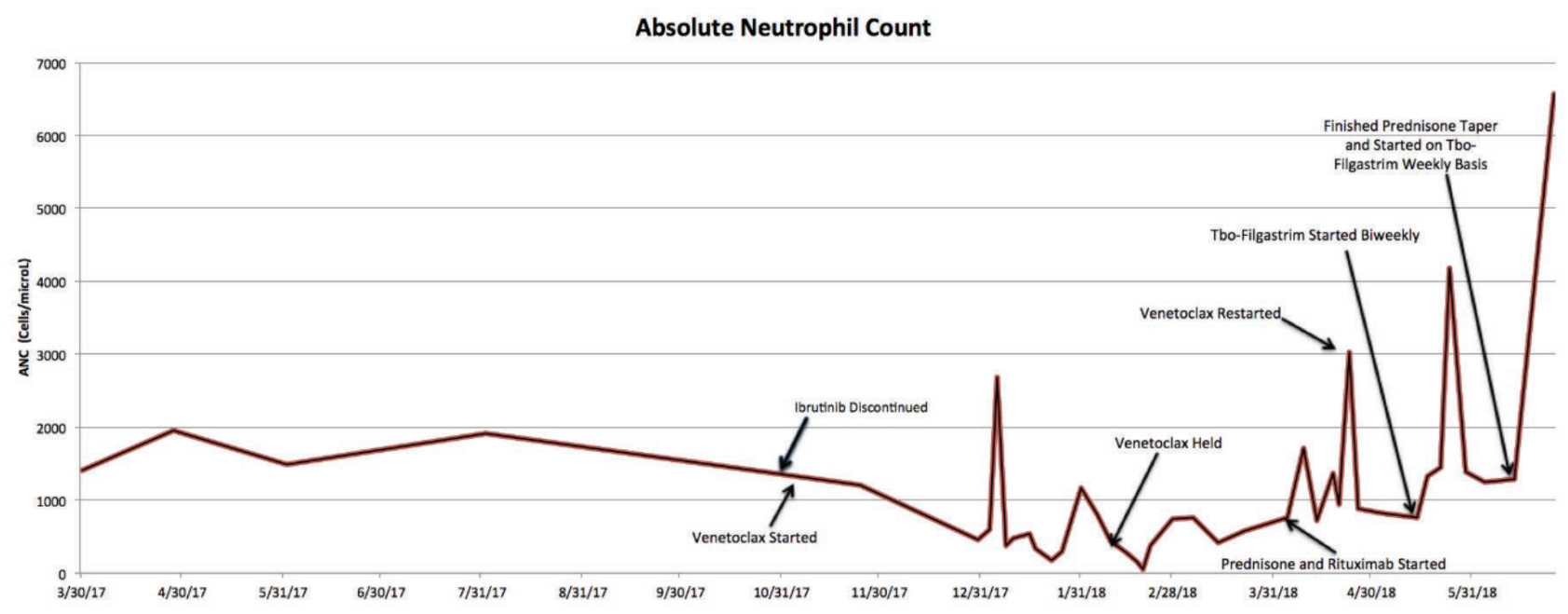

Figure 1.

\section{DISCUSSION}

We present a case of worsening AIN after stopping ibrutinib that was successfully managed with steroids, rituximab, and infrequent growth factor support. AIN is relatively uncommon in patients with $C L L$, but its presence should be suspected if other causes of neutropenia are ruled out. The pathogenesis of AICs in patients with CLL is not well understood and is possibly related to immune dysregulation including ineffective regulatory T-cell function. Additionally, humoral immunity can also be impacted as CLL cells may process cryptic antigens differently from normal B cells and thus present a peptide for which there is no immunological tolerance. ${ }^{6}$ Lastly, AICs in patients with CLL can also be drug-induced (purine analogs and alkylating agents).?

The role of ibrutinib in AIN is controversial and patients with AICs were largely excluded from pivotal ibrutinib clinical trials. In a retrospective analysis of $301 \mathrm{CLL}$ patients treated with ibrutinib at Ohio State University Cancer Center, six patients developed treatmentemergent AICs. However, these episodes of AIC occurred within 6 days to 23 months after ibrutinib initiation and thus did not appear to be temporally linked to the therapy. Furthermore, $25 \%$ of these patients had a past history of $\mathrm{AICs}$ and $7 \%$ were already receiving active therapy for $\mathrm{AIC}$ at the time of ibrutinib initiation. ${ }^{8}$

In the phase 3 RESONATE-1 study, which compared ibrutinib vs. ofatumumab in previously treated CLL/SLL patients, ibrutinib did not precipitate recurrent AICs in patients with CLL who had a history of autoimmune complications. ${ }^{9}$ In fact, ibrutinib may play a therapeutic role in the prevention and treatment of AlCs in patients with CLL. ${ }^{10}$ This was illustrated in one retrospective study using pooled data from four clinical trials which found that AIC therapy was discontinued in $86 \%$ of patients after a median of 4.7 months of initiating ibrutinib. ${ }^{8}$ Similarly, our group previously reported a case of CLL induced ITP that was successfully controlled with ibrutinib and rapidly flared after brief ibrutinib discontinuation. ${ }^{11}$ This also mirrors another case reported by Molica et al where a patient with CLL-associated AlHA experienced exacerbation of hemolysis twice after stopping ibrutinib.12 Conversely, some reports suggest that ibrutinib may initially and transiently worsen AlHA in patients with CLL, but with continued use, it may ultimately control AICs.

Our report suggests a role for ibrutinib in controlling CLL-associated AICs including AIN. Ibrutinib has a variety of mechanisms which can influence the immune system and potentially influence AICs. It inhibits interleukin-2inducible kinase (ITK), which decreases the response of autoreactive $T$ cells and can also modulate cytokine levels to promote a shift from a Th-2 towards a Th-1 mediated immune response. Additionally, ibrutinib inhibits B-cell receptor signaling which can inhibit the ability of B cells to respond to produce pathologic antibodies. ${ }^{8}$ Literature on this notion is currently conflicting and further research is needed to better elucidate the relationship between ibrutinib, CLL, and AICs.

CLL-associated AICs, such as AIN, are common throughout the course of disease. Steroids, immunosuppressive, or granulocyte stimulating therapy in the case of AIN are the current standard treatment options. Ibrutinib may be a new therapeutic option for AIN patients with concomitant treatment indications for their underlying CLL. This case also illustrates that some patients may develop an AIN flare after stopping ibrutinib. 


\section{REFERENCES}

1. Hodgson K, Ferrer G, Pereira A. Autoimmune cytopenia in chronic lymphocytic leukaemia: diagnosis and treatment. Br J Haematol 2011:154(1):14-22.

2. Hodgson K, Ferrer G, Pereira A, Moreno C, Montserrat E. Autoimmune cytopenia in chronic lymphocytic leukaemia: diagnosis and treatment. $\mathrm{Br}$ J Haematol 2011;154(1):14-22.

3. Visco C, Barcellini W, Maura F. Autoimmune cytopenias in chronic lymphocytic leukemia. Am J Hematol 2014;89(11):1055-62.

4. Klumpp TR, Herman JH, Schnell MK, Goldberg SL, Mangan KF. Association between antibodies reactive with neutrophils, rate of neutrophil engraftment, and incidence of post-engraftment neutropenia following BMT. Bone Marrow Transplant 1996:18(3):559-64

5. Byrd JC, Furman RR, Coutre SE. Three-year follow-up oftreatment-naive and previously treated patients with CLL andSLL receiving single-agent ibrutinib. Blood 2015:(125):2497-506

6. Dearden C. Disease-specific complications of chronic lymphocytic leukemia Hematology Am Soc Hematol Educ Program 2008:450-6.
7. Tandra P, Krishnamurthy J, Bhatt VR, Newman K, Armitage JO, Akhtari M. Autoimmune cytopenias in chronic lymphocytic leukemia, facts and myths Mediterr J Hematol Infect Dis 2013;5(1):e2013068.

8. Rogers KA, Ruppert AS, Bingman A, et al. Incidence and description of autoimmune cytopenias during treatment with ibrutinib for chronic lymphocytic leukemia. Leukemia 2016;30(2):346-50

9. Montillo M, O'Brien S, Tedeschi A, et al. Ibrutinib in previously treated chronic lymphocytic leukemia patients with autoimmune cytopenias in the RESONATE study. Blood Cancer J 2017;7(2):e524

10. Tsang M, Parikh SA. A concise review of autoimmune cytopenias in chronic lymphocytic leukemia. Curr Hematol Malig Rep 2017;12(1):29-38.

11. Sato R, Jacob J, Gaballa S. Rapid flare of immune thrombocytopenia afterstopping ibrutinib in a patient with chroniclymphocytic leukemia. Leuk Lymphoma 2017;

12. Molica S, Levato L, Mirabelli R. Chroniclymphocytic leukemia, autoimmune hemolytic anemia and ibrutinib: a case reportand review of the literature. Leuk Lymphoma 2016:57(3):735-7.

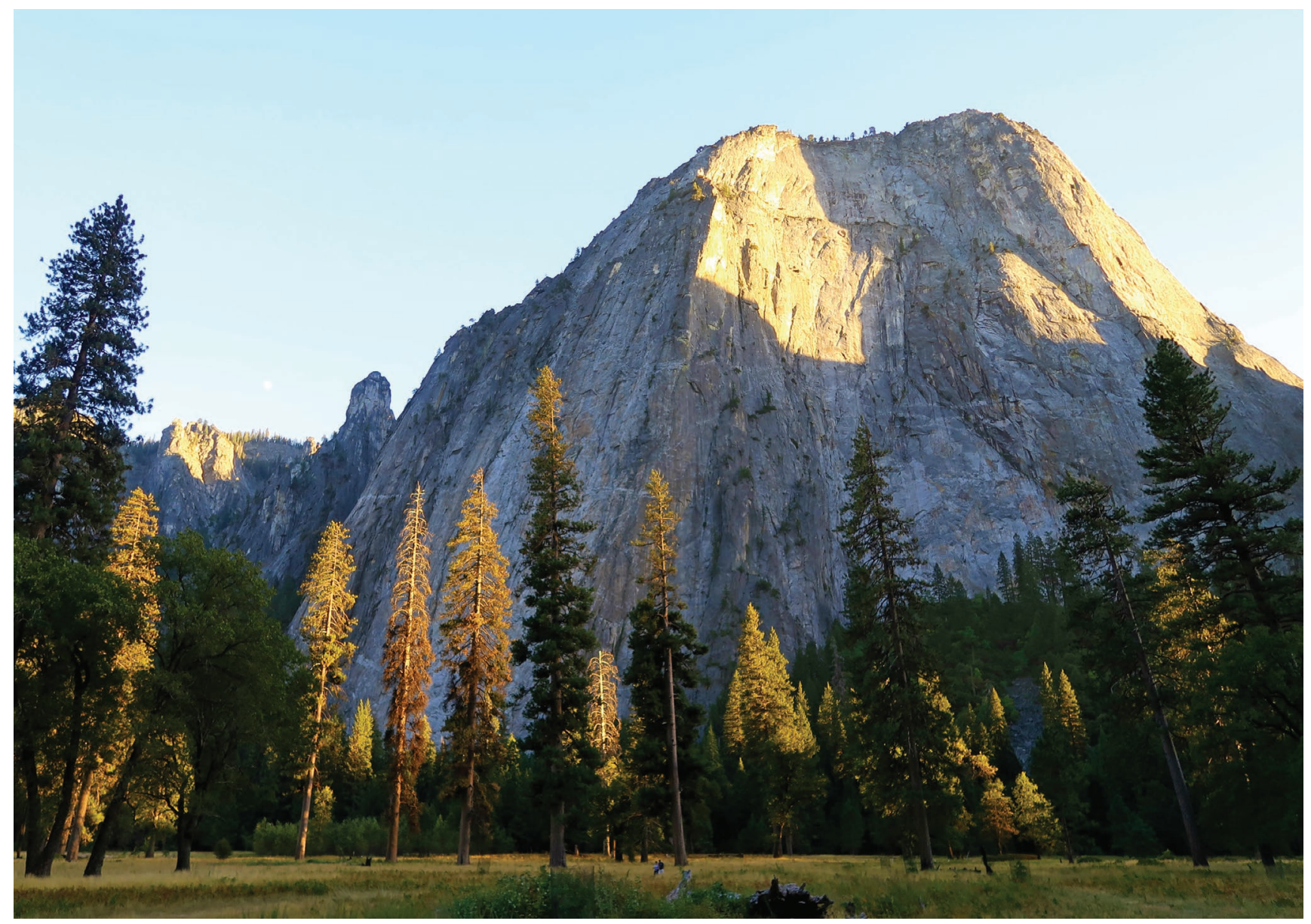

